\title{
Lumen
}

Selected Proceedings from the Canadian Society for Eighteenth-Century Studies

\section{Les résurgences des représentations catastrophiques de l'histoire à la fin du XVIII ${ }^{\mathrm{e}}$ siècle}

\section{Jean-Jacques Tatin-Gourier}

Volume 18, 1999

Representations of Time in the XVIIIth Century

Le temps et ses représentations au dix-huitième siècle

URI : https://id.erudit.org/iderudit/1012374ar

DOI : https://doi.org/10.7202/1012374ar

Aller au sommaire du numéro

Éditeur(s)

Canadian Society for Eighteenth-Century Studies / Société canadienne d'étude du dix-huitième siècle

ISSN

1209-3696 (imprimé)

1927-8284 (numérique)

Découvrir la revue

Citer cet article

Tatin-Gourier, J.-J. (1999). Les résurgences des représentations catastrophiques de l'histoire à la fin du XVIII ${ }^{\mathrm{e}}$ siècle. Lumen, 18, 175-184.

https://doi.org/10.7202/1012374ar

Copyright (C Canadian Society for Eighteenth-Century Studies / Sociéte canadienne d'étude du dix-huitième siècle, 1999
Ce document est protégé par la loi sur le droit d'auteur. L'utilisation des services d'Érudit (y compris la reproduction) est assujettie à sa politique d'utilisation que vous pouvez consulter en ligne.

https://apropos.erudit.org/fr/usagers/politique-dutilisation/ 


\section{Les résurgences des représentations catastrophiques de l'histoire à la fin du XVIIIe siècle}

Des Lettres anglaises de Voltaire (1734) au Tableau historique des progrès de l'esprit humain de Condorcet (1794), il n'est à aucun moment d'hégémonie de l'idée de progrès, contrairement à une pieuse imagerie 'progressiste' dont la constitution ne retiendra pas ici notre attention. En matière de représentations du devenir historique, le discours philosophique du midi du siècle est en fait parcouru de tensions et de failles. Pour Voltaire lui-même, les proclamations de foi dans le progrès visent d'abord à conjurer les résurgences toujours possibles des barbaries passées. Et que de textes enfin, où, loin des perspectives de progrès initiées par le Voltaire des Lettres anglaises, le lecteur est invité à se reporter vers une perfection des origines. Dans Le règne de l'histoire, Jean Marie Goulemot souligne la complexité des contiguités et des interférences vers 1750: '[1]e midi du siècle est le lieu d'une pluralité confuse qui donne à lire la pérennité des choses déjà dites, la cohabitation de l'ancien et du nouveau, les régressions et les articulations contradictoires. Aux tracés nets et distincts, il faut donc substituer l'image d'un enchevêtrement, d'un entrelacs minutieux et serré de rapports, de retours et de pénétrations. ${ }^{1}$ Ces ambiguités et ces tensions sont repérables dans les Discours de Rousseau et dans le 'Discours préliminaire' de l'Encyclopédie de D'Alembert, tout comme dans le De l'esprit d'Helvétius et dans le Code de la nature de Morelly, qui, si novateurs soient-ils en matière d'anthropologie, se fondent largement sur la représentation classique du devenir historique. Le second versant du siècle, et plus précisément les deux décennies qui précèdent le déclenchement du processus révolutionnaire, ne connaissent pas plus le 'triomphe' (selon l'expression convenue) de l'idée de progrès. Pour Rousseau, et plus encore pour un Diderot qui, à l'heure des bilans, ne se détourne nullement de la politique, il s'agit plutôt de prendre la mesure des illusions passées.

Il est, dans les Confessions, une étrange mise à distance de la profération du discours politique. Quand il évoque ses performances passées en cette matière, Rousseau a étonnamment recours au lexique du théâtre. Il n'est plus là question de vérité: Rousseau évoque un rôle tenu, des 
poses adoptées - et l'on connaît le regard porté par Rousseau sur la pratique du théâtre. Dans le cinquième livre des Confessions, Rousseau évoque la guerre du Piémont et les colonnes de l'armée française passant par Chambéry. Il souligne alors sa paradoxale 'partialité pour la France' qui, d'après lui, trouve son origine dans son goût pour la littérature française. Il suggère que ce penchant précoce est demeuré ensuite permanent envers et contre tout et que ses dénonciations ultérieures du despotisme n'ont fait que masquer, dans une sorte d'insincérité, cette vérité première:

Jusque-là je ne m'étais pas encore avisé de songer aux affaires publiques, et je me mis à lire les gazettes pour la première fois, mais avec une telle partialité pour la France que le cœur me battait de joie à ses moindres avantages et que ses revers m'affligeaient comme s'ils fussent tombés sur moi. Si cette folie n'eût été que passagère je ne daignerais pas en parler, mais elle s'est tellement enracinée dans mon cœur sans aucune raison, que lorsque j'ai fait dans la suite à Paris l'antidespote et le fier républicain, je sentais en dépit de moi-même une prédilection secrète pour cette même nation que je trouvais servile, et pour ce gouvernement que j'affectais de fronder. ${ }^{2}$

Les Confessions soulignent ainsi la vanité et la vacuité d'une contestation politique qui relèverait même de la falsification ('j'affectais de fronder'). Ce sont du même coup les perspectives d'un devoir-être politique, les fameux 'principes' (tels que les énonce le Contrat social), garants potentiels d'un avenir collectif juste et légitime, qui se trouvent frappés de suspicion: Jean-Jacques autobiographe semble reconnaître ici que, dans sa propre vie, ces principes proclamés n'ont eu d'autre fondement que le mensonge.

Les rêveries du promeneur solitaire réduisent plus encore toute perspective d'avenir: la lecture des Rêveries elles-mêmes est le seul avenir envisagé dans un texte où la proximité de la mort est constamment rappelée. De plus, Jean-Jacques y affirme le caractère absolu et irrémédiable de sa rupture de tout lien social: les persécutions constamment subies auraient tout à la fois effacé en lui la conscience de la primauté de l'intérêt général et toute possibilité de projection vers un avenir irrémédiablement fermé (toute possibilité de réhabilitation par la postérité est plus particulièrement niée):

Tant que les hommes furent mes frères, je me faisais des projets de félicité terrestre; ces projets étant toujours relatifs au tout, je ne pouvais être heureux que de la félicité publique et jamais l'idée d'un bonheur particulier n'a touché mon cœur que quand j'ai vu mes frères ne chercher le leur que dans ma misère. ${ }^{3}$ 
Après les persécutions, les condamnations venant de tous les horizons de l'opinion tant française que genevoise, il ne peut plus être que de difficiles et fragiles tentatives de restauration du rapport social: ultimes rencontres extasiées d'enfants, dialogue avec le vieil invalide qui passe la Seine et échappe à l'unanime prévention contre Jean-Jacques. Ce sont ces rencontres qui permettent à la fin des Rêveries de construire un étrange monde social épuré et évanescent, fondamentalement précaire et éphémère, loin des principes de la cité juste, définitivement reléguée dans un passé que la haine a détruit. Les figures du citoyen et du législateur s'estompent ainsi pour laisser la place à la figure du solitaire oscillant de l'abandon heureux aux sensations, à la méditation et aux ultimes rencontres tantôt porteuses d'effroi, tantôt brèves et émerveillées. Les rêveries du promeneur solitaire nous donnent ainsi à lire un au-delà de la politique et de ses espoirs: un monde en ce sens sans avenir.

Mais ce sont d'abord les œuvres de la vieillesse de Diderot (les Pages contre un tyran, l'Essai sur les règnes de Claude et de Néron, les Mélanges à Catherine II, les Observations sur le Nakaz et les contributions à l'Histoire philosophique des deux Indes de l'abbé Raynal) qui permettent de lire le plus nettement la dynamique d'une désillusion historique. Si Frédéric II, 'boutefeu de l'Europe', est très vite jugé et rejeté, il n'en est nullement de même pour Catherine II de Russie, en qui Diderot place d'abord tous ses espoirs d'une réforme philosophique, d'une refondation profonde de l'empire où l'impératrice 'forme[rait] elle-même une digue à la souveraineté. ${ }^{4}$ Cependant l'impatience et la crainte du temps perdu dans la guerre russo-turque cèdent vite la place à la déception et au constat amer de la permanence du despotisme au-delà des mises en garde et des conseils finalement vains du philosophe.

Les conseils et les souhaits adressés dans un premier temps à Catherine II impliquent une forte antithèse de la Russie et de la France, antithèse qui se fonde précisément sur le rapport des deux empires au devenir historique. La France intervient là très nettement comme repoussoir: ayant montré avec l'affaire du parlement Maupeou qu'elle était disposée à laisser détruire le fantôme même de sa liberté, ${ }^{5}$ elle n'est plus qu'un empire malade et sans avenir, parvenu au comble de son avilissement et de sa servitude:

[e]t puis je m'arrête pour considérer un moment par combien de vicissitudes nous avons été conduits au point où nous en sommes, ou plutôt où nous en étions, et par combien de vicissitudes nous aurions eu encore à passer pour arriver à quelque chose de bien, en continuant de nous abandonner aveuglément à ce mouvement obscur et sourd qui nous tiraille, qui nous tourmente et nous 
fait tourner et retourner, jusqu'à ce que nous ayons trouvé une position moins incommode, mouvement qui agite un empire mal policé, comme il agite un malade! Mais nous avons perdu jusqu'à cette inquiétude automate. Nous ne nous sentons plus. Il y avait dans le commencement un roi, des seigneurs et des serfs. Il n'y a aujourd'hui qu'un maître et des serfs sous toutes sortes de noms. ${ }^{6}$

Cette mise en perspective fondamentalement pessimiste du devenir historique du royaume (une marche inéluctable vers le despotisme) est dominée par la métaphore traditionnelle du corps malade, douloureux puis insensible, au terme d'une chute dont la proximité avec la mort n'a nul besoin d'être explicitée. Si hostile qu'il soit aux parlementaires, ces 'sots et orgueilleux remontrants', ${ }^{7}$ Diderot considère que la destruction des parlements par le pouvoir absolutiste a précipité une décadence aussi générale - elle concerne également la dynamique des connaissances - qu'irréversible:

[q]uand un peuple n'est pas libre, c'est encore une chose précieuse que l'opinion qu'il a de sa liberté; il avait cette opinion, il fallait la lui laisser; à présent il est esclave, et il le sent et il le voit; aussi n'en attendez plus rien de grand ni à la guerre, ni dans les sciences, ni dans les lettres, ni dans les arts. La philosophie est persécutée. Les lettres ne se soutiennent que par la considération publique d'un peuple qui s'ennuie et qui ne peut refuser sa faveur à des hommes qui l'amusent; il n'y a que du danger à écrire et penser hardiment. On ne peut recueillir de son ouvrage aucun lucre, aucun honneur, parce qu'on ne peut l'avouer. Ce sentiment patriotique vit encore dans les pères; il vit même au fond des cœurs de tous les fauteurs actuels de la tyrannie; et c'est par cette raison qu'on n'ose pas tout contre les pères qu'on ne croit pas disposés à tout supporter. Mais les successeurs de ces ministres de la tyrannie seront des tigres qui se croiront nés de tout temps pour être déchirés.

$\mathrm{O}$ nation si belle, il n'y a qu'un moment! $\mathrm{O}$ malheureuse nation, je ne puis m'empêcher de pleurer sur toi!

Il est une haute montagne, escarpée d'un côté et terminée de l'autre par un précipice profond; entre le côté escarpé et le précipice il y a une plaine plus ou moins étendue. La nation qui naît grimpe le côté escarpé. La nation formée se promène sur la plaine. La nation qui déchoit suit la pente du précipice, et la suit avec une grande célérité; nous y sommes. ${ }^{8}$

A la métaphore de la décrépitude physique succède donc celle de la chute. L'abîme n'est toutefois pas un terme pour Diderot qui envisage un au-delà de la décadence sur un ton prophétique assuré: il est 'des révolutions qui surviennent toujours à l'extrémité du malheur' par delà lesquelles le philosophe qui a inlassablement 'éclairé les hommes sur leurs droits inaliénables' peut envisager 'des suites qui compensent le 
sang répandu. ${ }^{9} \mathrm{Si}$ inconnu soit-il, cet avenir est porté par l'intelligence du philosophe et parallèle à la sourde poussée de forces sociales en devenir: ' $[t]$ out cela se peut et se fera un jour. Sans compter la progression du tiers état qui ira toujours sourdement. ${ }^{, 10}$

Mais ce ressurgissement de perspectives positives, si assurée qu'en soit la prévision, demeure flou et largement indéterminé sur le plan temporel. Il est de plus une appréhension tout autre - foncièrement pessimiste - de cet avenir qui se dérobe décidément à toute appréhension en termes de progrès. C'est ce qu'atteste, toujours dans les Mélanges à Catherine II, le rêve burlesque du règne du 'roi Denis', qui tente de 'rendre les mœurs, l'éclat et la vie à sa pauvre nation, ou [de] faire renaître une autre sorte de luxe qui ne soit plus le masque de la misère, mais le signe de l'aisance publique et du bonheur général. ${ }^{11}$ Mais ce rêve se clôt sur la perspective d'une révolution destructrice mettant un terme à la vie et à l'œuvre du monarque réformateur:

[e]t puis, quand Denis passe dans les rues de la capitale, c'est un tumulte, un bruit, des acclamations, des Vive Denis! qui ne finissent point; et puis Denis, qui a l'âme tendre, s'élance de son carrosse, on l'embrasse; il est embrassé sur le Pont-Neuf comme Catherine Seconde l'est dans son couvent et le sera un jour dans les rues, et puis il meurt doucement, regretté, pleuré, honoré; ou bien on le tue et il meurt violemment. Et qu'est-ce que cela fait? Il n'en est ni plus ni moins mort. $^{12}$

Dans les Mélanges à Catherine II, Diderot promet à la Russie un avenir brillant que garantissent les réformes hardies de la souveraine. Diderot rappelle cependant que l'œuvre entreprise par Catherine n'est qu'une consolidation: si parfait qu'il soit, l'ouvrage ne peut échapper à l'érosion du temps:

[...] il a fallu des siècles pour amener notre instant fatal; et cet instant pouvait être retardé par des lois et des institutions sages, si nous en avions eu. Songez, madame, que je vous présente l'éboulement d'un grand amas de grains de sable que des circonstances fortuites avaient entassés, au lieu qu'il dépend de Votre Majesté de placer la base de votre pyramide sur le roc, et d'en lier les différentes parties par des crampons de fer. Le roc s'affaisse, il est vrai, les crampons de fer se relâchent, les pierres se disjoignent, et l'édifice s'écroule à la longue; mais il a duré cent siècles; cent siècles d'un bonheur continu et procuré par les travaux et le génie étonnant de Votre Majesté à trente millions d'hommes, ne suffiront-ils pas à son âme vaste et grande? ${ }^{13}$

A la fin des fins, l'antithèse de la France au bord de l'abîme et de la Russie à la veille de sa régénération tend donc à s'effacer: tout comme 
un penseur de l'âge classique, Diderot n'envisage que la mort comme horizon des empires, et l'art de gouverner n'est pour lui qu'une manière de retarder cette inéluctable fin. Les écrits politiques de Diderot attestent ainsi que la problématique de l'absolutisme éclairé se fonde pour une large part sur des représentations fort anciennes du devenir historique.

Écrites peu de temps après les Mélanges et le retour de Saint-Pétersbourg, les Observations sur le Nakaz (1774) proclament le caractère illusoire, en Russie comme dans tous les pays d'Europe, de tout espoir en un avenir que transformerait un souverain docile aux leçons de la philosophie. Diderot souligne l'écart tragique entre les vérités politiques acquises par l'homme opprimé mais se servant de sa raison, et les réalités politiques de fait caractérisées par un renforcement sans précédent du despotisme:

Toutes ces idées si justes, si raisonnables, que les membres ne furent pas faits par le chef, mais le chef par les membres, qu'il y a un pacte tacite, des droits inaliénables, une liberté, une propriété, sont bien nouvelles relativement à l'institution première de la société. C'est le cri de l'homme opprimé, c'est le produit d'une longue suite de maux éprouvés par l'abus de l'autorité. Alors la raison était bien avancée, lorsque l'homme se demanda ce que c'était qu'un homme, l'individu ce que c'était qu'un souverain. Les lumières sur tous ces points ont été poussées de nos jours aussi loin qu'il était possible. Qu'ont-elles produit? Rien; au milieu de la réclamation de tous les peuples civilisés par la voix de magistrats et des philosophes, le despotisme s'étend de tous les côtés. Nous sommes encore bien loin du moment où l'on lira à la tête d'un édit: "Louis, Frédéric, Catherine par la grâce de ses sujets" et non "par la grâce de Dieu"; cette innovation immortalisera le premier souverain qui la fera. ${ }^{14}$

Au-delà de ce constat désespéré de l'inefficacité politique des Lumières, il n'est plus de place que pour la prophétie d'un avenir réparateur aussi lointain que vague: '[u]n temps viendra que toutes les iniquités seront citées au tribunal des nations et que la puissance qui les commet sera elle-même jugée par ses victimes. ${ }^{15}$

Dans les ultimes textes politiques de Diderot, il est en fait une fréquente oscillation du constat d'un avenir irrémédiablement fermé à l'affirmation au ton messianique d'un avenir indéterminé de justice réparatrice. Le lecteur ne peut trancher: les temps présents constituent-ils '[la barbarie] d'un peuple qui n'a pas encore les yeux ouverts' ou celle 'd'un peuple qui a les yeux crevés', 'la barbarie dont [la nation] ne sort plus?'16

L'Essai sur les règnes de Claude et de Néron (1779) fait indéniablement pencher la balance dans le sens du pessimisme. Dans cette fresque crépusculaire des plus atroces déchaînements du despotisme, Diderot 
restitue, à l'heure de ses propres bilans, l'action désespérée de Sénèque, du philosophe conseiller d'un prince, dont il a été auparavant l'éducateur, sombrant dans une folie monstrueuse et sanguinaire sans précédent dans l'histoire. Action de plus en plus désespérée face à un avenir qui s'assombrit puis se ferme dans un enchaînement de crimes sans fin qui constitue une véritable marche à l'abîme: assassinats de Messaline, d'Agrippine, de Sénèque enfin; incendie criminel d'une Rome minée par la corruption et la superstition:

L'incendie dura six jours et sept nuits. Néron, spectateur du haut de la tour de Mécène, en habit de théâtre, chante l'embrasement de Troie. Il défend de fouiller les décombres: on en tire à son profit les restes de la fortune des incendiés; et pour la réparation du désastre, il exige des contributions qui ruinent la ville et les provinces. Il dit: "Faisons en sorte que tout m'appartienne". L'indiscrétion d'un souverain laisse quelquefois échapper la secrète pensée des autres; ils se taisent mais leurs vexations parlent. ${ }^{17}$

Par sa renommée auprès du public, Sénèque était prédisposé à être manipulé, instrumentalisé par un despote habile à dissimuler son despotisme. Le philosophe s'est en fait pris au double piège du pouvoir et de l'opinion:

Mais le règne de Claude s'échappe; la scène va changer, et nous montrer le philosophe Sénèque à côté du plus méchant des princes, dans la cruelle alternative ou d'encourir le soupçon de pusillanimité, d'avarice, d'ambition, de vanité, s'il reste à la cour; ou le reproche d'avoir manqué à son élève, à son prince, à sa patrie, à son devoir, et sacrifié inutilement sa vie, s'il s'éloigne. Quelque parti qu'il prenne, il sera blâmé. ${ }^{18}$

En acceptant les premières dignités de l'empire, Sénèque a néanmoins fait œuvre de 'résistance' et empêché la multiplication immédiate des crimes de Néron:

Sénèque, qu'as-tu fait de Néron?

- J'en ai fait tout ce qu'il était possible d'en faire. J'ai emmuselé l'animal féroce; sans moi, il eût dévoré cinq ans plus tôt. ${ }^{19}$

Au-delà des ambiguités auxquelles il a été contraint par le plus pervers des despotes, Sénèque a su ne pas confondre les rôles de courtisan et de philosophe. Sa mort héroïque, comparable à celle de Socrate, atteste cette intégrité. Les figures de Néron et de Sénèque sont bien évidemment emblématiques: 
[c]e n'est pas sous un Tibère, sous un Néron seulement, c'est de tous les temps et dans toutes les cours qu'il y a plus de faveur à se promettre du métier de proxénète que des fonctions de grand ministre, et quel'on peut sans conséquence déshonorer une nation par la perte d'une bataille, mais non adresser un mot ou un geste de mépris à une favorite. ${ }^{20}$

Au travers de la justification de Sénèque tentant de tenir jusqu'au bout aux côtés du despote pour limiter ses crimes se profilent des situations contemporaines brièvement mais nettement évoquées: le despotisme de la fin du règne de Louis $X V$, les renvois de Malesherbes et de Turgot, une situation où 'l'esprit philosophique' se refuse décidément à 's'introduire dans l'histoire. ${ }^{21}$ Quant aux sursauts populaires contre le despotisme, il ne faut selon Diderot rien en attendre. Ils ne peuvent en effet que précipiter l'agonie de l'empire: les esclaves sont 'sans vues, sans principes, sans plans', 'ils ne savent comment assurer leur bonheur à venir. ${ }^{22}$ L'horizon de la sédition ne peut être que la résurgence du despotisme.

Ainsi, dès 1779 , l'Essai sur les règnes de Claude et de Néron marque bien pour Diderot la fin des espoirs en une monarchie éclairée. Après l'effondrement du modèle russe dès 1774 et les ultimes désillusions françaises des premières années du règne de Louis XVI, le détour par l'histoire romaine s'impose pour dénoncer l'illusion politique de toute une vie et pour dire la hantise de la fin inexorable des empires. Cette hantise est aussi, indistinctement, celle d'une fin des Lumières: Diderot souligne qu'avant même la mort de Sénèque, les charlatans, usurpant la place du philosophe, s'imposent et prolifèrent dans la Rome superstitieuse et corrompue de Néron. 'Rome alors était pleine d'astrologues et de diseurs de bonne aventure $[\ldots]^{.23}$

Le développement du mesmérisme et plus encore le scandale de la fascination exercée par Cagliostro ${ }^{24}$ sur les élites parisiennes redoubleront, peu avant la Révolution, la crainte d'une hégémonie du 'charlatanisme moderne' prenant le pas définitivement sur la philosophie véritable. Les volumes d'Economie politique et diplomatique de l'Encyclopédie méthodique, vaste entreprise dirigée par le libraire Panckoucke qui prétend, au début des années 1780, reprendre le flambeau de Diderot, manifestent un pessimisme politique absolu en même temps qu'ils exigent de la part des gouvernants la plus grande prudence en matière de réformes: ${ }^{25}$

[1]es Etats, ainsi que les corps humains, portent en eux les germes de leur destruction: comme eux, ils jouissent d'une force plus ou moins durable; comme eux, ils sont sujets à des crises qui les enlèvent brusquement, ou à des maladies chroniques qui les minent peu à peu, en attaquant les principes de la vie. Ainsi les sociétés, comme les malades, éprouvent des transports, des délires, des 
révolutions: un embonpoint trompeur couvre souvent leurs maladies internes; la mort elle-même suit de près la santé la plus robuste. [...] Un usage affermi par le temps, utile ou non, est pourtant à sa place dans l'enchaînement des choses; tout est si bien lié, que la moindre nouveauté substituée aux abus courants, ne tiendra jamais à la tissure comme une partie usée; et tel changement serait bon en lui-même, qui gâterait tout par la difficulté de l'assortir au reste. ${ }^{26}$

Ainsi, bien au-delà de la vieillesse des philosophes, à la veille même de la Révolution française, les visions catastrophiques de l'histoire resurgissent massivement chez les disciples des encyclopédistes.

\section{JEAN-JACQUES TATIN-GOURIER}

Université F. Rabelais de Tours

\section{Notes}

1 Jean Marie Goulemot, Le règne de l'histoire (Paris:Albin Michel, 1997) 346.

2 Ed. B. Gagnebin et M. Raymond (Paris: Gallimard, 1959) I, 182.

3 Ibid., 1066.

4 Diderot, Mélanges à Catherine II, dans CEuvres complètes (Paris: Robert Laffont, 1994) III, 209.

5 ' [...] toutes ses résistances aux volontés du souverain n'étaient que de la mômerie; [...] l'intérêt de la nation était toujours sacrifié et [...] il [le parlement] ne se battait bravement que pour le sien' (Mélanges, 21).

6 Ibid., 212.

7 Ibid., 214.

8 Ibid., 216-17.

9 Ibid., 348.

10 Ibid., 360.

11 Ibid., 295.

12 Ibid., 301.

13 Ibid., 217.

14 Diderot, Observations sur le Nakaz (CEuvres complètes, 546).

15 Ibid., 541-42.

16 Ibid., 571.

17 CEuvres complètes, I, 1058.

18 Ibid., 1000.

19 Ibid., 1012. 


\section{Jean-Jacques Tatin-Gourier}

20 Ibid., 1054.

21 Ibid., 1202.

22 Ibid., 987.

23 Ibid., 1063.

24 Cf. Jean-Jacques Tatin-Gourier, Cagliostro et l'affaire du Collier (Saint Étienne: Publications de l'Université de Saint-Etienne, 1994).

25 Les quatre volumes d'Economie politique et diplomatique, publiés en 1788, sont l'œuvre de Jean-Nicolas Desmeunier (1751-181 ) et de Guillaume Grivel (1735-1810).

26 Economie politique et diplomatique, III, article «Politique». 